\title{
Analysis on the 5W Model of MUZEN RADIO Brand Communication in the New Media Environment
}

\author{
Lu Wang ${ }^{1, *}$ Qiuyan Yun ${ }^{1}$ \\ ${ }^{1}$ College of Arts and Sciences, Shanghai Polytechnic University, Shanghai 201209, China \\ *Corresponding author.Email:15658908@qq.com
}

\begin{abstract}
At present, as one of the retro audio brands, MUZEN RADIO is very popular among young people. This article draws on Laswell's 5W model, from the aspects of communicator (who), communication content (what), communication channel (in which channel), audience (whom) and communication effect (with what effect), etc., to carry out in-depth analysis of the brand communication model of MUZEN RADIO, trying to show the detailed picture of the brand communication of MUZEN RADIO in the new media environment. And on this basis, this article puts forward corresponding suggestions on the deficiencies in the brand communication of MUZEN RADIO, in order to provide reference and reference for the development of Chinese audio brand communication.
\end{abstract}

Keywords: New media, 5W model, MUZEN RADIO.

\section{INTRODUCTION}

Brand communication is a process in which brand owners use a variety of communication methods to continuously display and express brand elements and spread the superior value of the brand in order to maintain or enhance audiences' reputation and loyalty to their brand. As one of the retro audio brands, MUZEN RADIO has been loved by young people in recent years. This article draws on Laswell's famous "5W" model, namely the communicator (who), communication content (what), communication channel (in which channel), audience (whom) and communication effect (with what effect), which can three-dimensionally depict the spread of MUZEN RADIO in the new media environment. [1]

*Project: This article is the research result of the Public Relations Discipline Construction Project of Shanghai Polytechnic University "New Media Public Relations" (discipline number: XXKPY 1610); this article is the related research result of Shanghai Polytechnic University's 2019 Excellent Course Construction "Organizational Culture" ( A01GY20GO13-04).

\section{ANALYSIS OF BRAND COMMUNICATION ELEMENTS OF MUZEN RADIO}

\subsection{Communicator (Who)}

\subsubsection{Oral Statement by Product Purchasers}

Purchasers of MUZEN RADIO products will have a different product experience after using the product. When others need to buy audio products, the purchasers will dictate their product experience. Product purchasers belong to the purchaser group, and the oral method does not rely on any media, which is different from the recommendations of the merchants, and has higher authenticity and can give the audiences a sense of trust. In the brand, audiences who purchase the product advertise the product to other audiences, and when other audiences make purchases, secondary communication can be formed. 


\subsubsection{Recommendations from Offline Store Staff}

In May 2015, MUZEN RADIO began to expand offline retail stores. Among the physical stores of life aesthetics, the only audio products are from the MUZEN RADIO brand, and the product competition is relatively small. After entering the store, when the clerk learns that you have audio purchase needs, they will enthusiastically introduce the product. When introducing the product, the clerk will not only dictate, but also use body language to display the use of the product. Purchasers will also generate timely feedback. Offline clerk belongs to the seller group. As a direct communicator, face-to-face communication can make the information spread more effectively.

\subsubsection{The Guidance of Opinion Leaders}

In the information society, opinion leaders who rely on oral communication do not exist. The realtime nature of information dissemination makes opinion leaders have to resort to the media for mass communication, so the group of opinion leaders belongs to indirect communicators. The popular opinion leaders who appeared in the brand promotion of MUZEN RADIO were mainly Luo Zhenyu and Wu Xiaobo. They praised the brand's products and shifted the audience's attention to the brand's products. In addition, as the celebrity spokesperson of MUZEN RADIO, Cheer Chen is also one of the opinion leaders. She attended the product launch conference and filmed advertising videos. In addition to the guidance of popular opinion leaders, some niche opinion leaders can also be seen on platforms such as Little Red Book and Weibo. They share their own experience to achieve the effect of word-of-mouth communication and make more people pay attention to the brand.

\subsection{Communication Content (What)}

\subsubsection{Features of the Products}

The essence of word-of-mouth communication is to return to audiences' trust in products, so the importance of product quality is self-evident. [2]MUZEN RADIO is an exquisite and fashionable retro audio brand founded by Chinese audio designer Mr. Zeng Dejun. The products are made by hand. The overall sound adjustment is not the ultimate $\mathrm{HiFi}$ three-frequency clear route. In addition, MUZEN RADIO has a unique self- created APP, OhPlay, including exclusive broadcasts of MUZEN Music Channel, MUZEN Selected Radio, and integrated cross-platform free radio programs to meet people's diverse music needs.

\subsubsection{Brand Culture}

In this era where content is king, in addition to product quality, the most important thing is brand culture. [3]The brand culture of MUZEN RADIO is counter-mainstream and classic. The brand IP reflects a sense of happiness of small fortune.

It has always upheld the humanistic feelings of warmth, adhered to the value of "hear the difference", and is committed to letting people living in the Internet age can hear the joy of life.[4]

\subsection{Communication Channel: Online and Offline Linkage (in Which Channel)}

\subsubsection{Online Promotion}

\subsubsection{Multimedia-composite Promotion}

As the medium for communicating information, the wider the channel, the greater the possibility that the audiences will receive the information. MUZEN RADIO uses a multimedia-composite promotion method, covering Weibo, WeChat, Q\&A, Baidu, We-media and other platforms, including official accounts, advertising resources, Baidu Baike and other content. Online, in addition to Tmall and JD.com, MUZEN RADIO has also entered a large number of vertical content ecommerce businesses on the head and waist, including Yitiao, Wu Xiaobo Channel, Luo Ji Thinking, Ten O'clock Reading, etc., covering a variety of terminals including mobile phones and computers.[5]

\subsubsection{Interactive Communication on Social Platforms}

In the era of integrated media, information communication has changed from simple "text + pictures" to "video + music", enriching the types of communication forms.[6]The brand communication of MUZEN RADIO is advertised on various short video social platforms. In addition to the official video of MUZEN, there are also purchasers' product displays. Audiences interested in the product will interact with purchasers who have already purchased the product. 


\subsubsection{Offline Promotion}

\subsubsection{Physical Stores}

Many brands of smart speakers are sold in electrical appliance chains, but MUZEN RADIO, in contrast, chooses not to enter offline electric appliance chains. In May 2015, MUZEN RADIO began to expand offline retail stores. The most important point for store selection is tonal matching. Lifestyle chain brands that have settled in shopping malls, such as the home electric appliance mall Shundian, the electric appliance firm Sound Town, and the bookstore Yanjiyou, which focuses on the aesthetics of life. The clerk in the store, as a communicator, when recommending the Little Prince, they will say that it uses a new aluminum alloy material and has been painted three times with car paint, so it's something elastic and breathable. The audiences as audiences will receive the message, feel its brand culture, and feel its value.

\subsubsection{Experience Activity Meetings}

Another aspect that distinguishes MUZEN RADIO from other radios is the hosting of Radio Easter. In 2017, it launched the "Radio Easter" activity, gathering 15 well-known radio DJs from around the world to the Sahara Desert for a 24-hour non-stop radio broadcast. The number of online listeners exceeded 5.1 million, breaking the live record of QQ Music Radio. Many cross-border media people, such as Rebecca's Whimsical World, Poison Tongue Movies, bookface and other new type of self-media representatives with public appeal and influence, have joined the Radio Easter. The diversification of communication channels helps brands to promote unique brand characteristics, so that the audiences can better remember the MUZEN brand.

\subsection{Communication Audiences (Whom)}

Before 2014, the audiences of MUZEN RADIO were around 65 years old. However, with the integration with the market, the appearance and design of products tend to be younger and fashionable, and the age group of the audiences has changed, down to 15-35 years old. Due to changes in audience's selection, its sales have gone from 300 units in 2014 to over 1 million units in 2018.

In the new media environment, the audiences of MUZEN RADIO have changed. The original audiences of 15-35 years old have changed to audiences of young women, becoming the main force of the brand's increasing sales. In 2015, through Internet marketing and overall repackaging, the annual sales rose from 300 to 10,000 . In the past, the $3 \mathrm{C}$ electronic digital brand did not particularly consider the preferences of female audiences. Long ago, the brand had some colors specifically designed for women or launched for women, which were more personalized.

\subsection{Communication Effect (with What Effect)}

\subsubsection{Concerning Level: Paying Attention to the Brand}

The brand communication effect of MUZEN RADIO is first to gain the attention of the audiences.[7] From the perspective of the brand's communication channels, audiences aged 15-35 have wider exposure to media platforms and social platforms, and are more likely to receive information. However, due to the uniqueness of the product design, it is more likely to receive attention from female audiences. Only when a product gets the attention of the audiences can the they better understand the product itself and arouse emotional resonance.

\subsubsection{Emotional Level: Arousing Humanistic Feelings and Emotional Resonance}

The brand culture of MUZEN RADIO reflects the counter-mainstream niche culture. Each product has a different brand story, and each story is based on the radio culture. Whether it is the trendy culture or the brand story behind the product, it is mixed with emotional elements. After the audiences pay attention to the product, there will be emotional fluctuations and even arouse the resonance of humanistic feelings.

\subsubsection{Action Level: Generating Consumption Impulse}

After the audiences receive the message transmitted by the media, they will evoke emotional memory or recognition of the brand culture, and the design of the product will generate a audience impulse within a certain period of time. There will be a special layout scene in the offline physical store of MUZEN RADIO, which makes people feel warm and in line with its warm humanistic feelings. The advertisements on MUZEN RADIO in the 
store, the consumption scene created by POP, and the display of MUZEN products by the clerk, make the audiences in the store have an impulsive desire to consume, and finally achieve the behavior of instant consumption.

\section{DEFICIENCIES IN THE BRAND COMMUNICATION OF THE MUZEN RADIO}

By analyzing the brand communication elements of MUZEN RADIO, it can be found that in the new media environment, although the brand communication of MUZEN RADIO has its own characteristics, there are still shortcomings. The following will analyze each element layer by layer.[8]

\subsection{Communicator (Who)}

\subsubsection{Limitations of Word-of-mouth Communication}

There are limitations in word-of-mouth communication, and there are uncertain factors in the content of the communication, which leads to the unstable result of the final communication effect. When direct communicators express the experience of using MUZEN RADIO products to their audiences, the oral method obviously lacks many advantages compared with offline physical stores. They cannot allow the audiences to directly experience the products and cannot feel the warm atmosphere of the MUZEN RADIO counter in the store, etc., leading that the final communication effect cannot reach the best state.

\subsubsection{The Instability of the Fan Economy}

With the rapid development of the Internet, the fan economy has become an important part of the cultural industry market. The brand spokesperson of MUZEN RADIO is Cheer Chen. The number of fans on Weibo is only more than 410 thousands. Compared with other celebrities, the influence is not big, but it fits the tone of Elvis Xiaodou. The purpose of the fan economy is to hope customers will remain loyal and purchase repeatedly. However, MUZEN RADIO's products are an emotional economy that generates consumption by arousing the emotions of the audiences. Once the love disappears, the fan economy will cease to exist. [9]

\subsection{Communication Content (What)}

First of all, as the original app of MUZEN RADIO, OhPlay has advertisements on the content before the update, and the content is not the best quality. The existence of advertising shows that OhPlay does not strictly supervise radio stations, which leads to a poor experience of the radio audiences. There are two main functions of the MUZEN RADIO as a speaker. One is to play as a player, and the other is to connect to OhPlay to experience the unique charm of the radio. In the early publicity, MUZEN prestigiously focused on radio, with a relatively single coverage, which caused some confusion to the audiences.

Secondly, on platforms such as Little Red Book and Weibo, people can see many soft articles about MUZEN RADIO released by KOLs (key opinion leaders), most of which highlight the appearance and use characteristics of MUZEN RADIO. There are not many innovations in the propaganda content, mainly emotional soft articles, and the lack of personality causes the repetition of the articles, which leads to the selective attention of the audiences.

In addition, MUZEN RADIO focuses on brand culture when promoting its selling points, thus ignoring the promotion of product features. Purely handcrafted, the overall sound adjustment is not the ultimate $\mathrm{HiFi}$ three-frequency clear route. As the main feature of MUZEN RADIO, it is also an aspect of the audiences' attention. However, MUZEN has weakened the characteristics of the product as a whole during the promotion, so that the audiences cannot fully understand the characteristics of the product.

\subsection{Communication Channel (in Which Channel)}

\subsubsection{Simple Offline Media Communication}

The communication of MUZEN offline is mainly through the hosting of the Radio Easter, which has only been held in recent years. In addition to the DJ of the radio station, offline participants will also have media participation, and will reflect the situation of the event to the audiences in the form of tweets. But it has only been held three times so far, and the coverage of the content is relatively simple, and the audiences may not be able to see the relevant tweets. On the other hand, the Radio Cultural Festival focuses on radio 
culture, rarely introduces products, and the channels for product communication are relatively simple.

\subsubsection{Fewer Offline Physical Stores}

The reduction of offline sales channels will result in a corresponding reduction in brand communication channels. MUZEN RADIO CMO's(chief marketing officer) strategy is not to enter offline electrical appliance chains. Beginning in the second half of 2016, MUZEN cut some channels that did not meet the requirements. The Electronic City and Computer City basically disappeared from the list of cooperation, resulting in fewer options for offline stores.

\subsection{Communication Audiences (Whom)}

\subsubsection{The Communication Audiences Are Mainly Young People and the Scope Is Small}

The audiences of MUZEN RADIO have changed from 65 years old to 15-35 years old now. The age range of the audiences has changed and the range has been expanded, with female audiences as the main force. The product design is also dominated by female audiences, ignoring the needs of male audiences. According to the number of downloads of smart apps on the market, male users accounted for $60.5 \%$ and female users accounted for $39.5 \%$. In addition to the female audiences of MUZEN RADIO in this age group, there is still room for development in the male audiences market.

\subsubsection{Insufficient Brand Loyalty}

As the core element of brand equity, brand loyalty can not only effectively reduce the marketing costs of enterprises, but also can obtain good word-of-mouth communication through repeated purchases by more audiences, thereby continuously bringing new audiences. The first thing to maintain brand loyalty is to establish a distinctive brand culture. MUZEN RADIO has a unique brand culture - anti-mainstream and classic style. The second is to continue to interact with the audiences and become friends with them. MUZEN RADIO does not do enough in this part. There are too many soft texts on various media platforms and less online interaction. Offline radio cultural festivals are held once a year in recent years. It is difficult for audiences to maintain loyalty to the brand.

\subsection{Communication Effect (with What Effect)}

The brand culture of MUZEN RADIO is antimainstream and classic culture, projecting a sense of happiness, and its brand spokesperson is Cheer Chen. Regardless of the brand culture of MUZEN or the design of its products, it is mainly female audiences with relatively small coverage. Male audiences seldom recognize the sound that brings happiness and lack the brand identity of MUZEN. They pay more attention to the quality of products and the sense of technology in the future. Therefore, the brand culture of Elvis Radio restricts male audiences, and it is not very effective in communicating with male audiences.[10]

\section{SUGGESTIONS ON BRAND COMMUNICATION OF MUZEN RADIO}

\subsection{Communicator}

In the new media environment, people are more inclined to socialize or share information on social media. Word of mouth has gradually become the focus of attention, which brings unlimited business opportunities. The word-of-mouth communication of MUZEN RADIO mainly relies on two groups of product purchasers and opinion leaders. But on the media platform, MUZEN' opinion leaders tend to publish soft texts and lack interaction. The concept of social interaction and word-of-mouth allows users who have not clearly purchased a certain kind of radio to learn about MUZEN RADIO by sharing their purchase experience under the leadership of opinion leaders, which helps promote audiences to pay attention to MUZEN.

In addition, from previous studies, it can be found that the influence of MUZEN RADIO on social platforms is not prominent, and it is more of the sharing posts of purchasers. Therefore, MUZEN RADIO can regularly select opinion leaders who conform to the tone on different media platforms for publicity. 


\subsection{Communication Content}

\subsubsection{Strictly Controlling the Content Review of the Original Platform to Avoid Social Sensitive Issues}

MUZEN RADIO's unique APP, OhPlay, allows users to become radio anchors after applying, and the entire application process is relatively smooth. However, the lack of strict supervision will lead to many problems. For example, the radio content occasionally plays some uncopyrighted songs, uses uncivilized language and other behaviors, which will bring serious adverse effects to MUZEN RADIO. In addition, it is a must to pay attention to the supervision of the quality of radio anchors, try to select some positive radio anchors, and achieve strict content control.

\subsubsection{Strengthening the Innovation of Original Content}

Most of the OhPlay radio stations are mainly sound transmission, and the content is less different from the content of the FM APPs on the market, and there are fewer innovative programs. MUZEN can use big data to collect user information in the background, understand audience preferences, produce radio programs that satisfy the public based on the data, and then innovate the content of the programs. In addition, it is necessary to pay attention to the promotion of MUZEN RADIO's "little fortunate happiness", moving people with emotion, rather than just imitating others and failing to innovate.

\subsection{Communication Channel}

In the era of new media, traditional mass communication has been slowly broken, the media has begun to diversify, and absolutely efficient forms of media communication no longer exist. Therefore, diversified brand communication methods have become more and more important. MUZEN RADIO has many communication platforms, such as Zhihu, Weibo, Douyin, etc., but lacks regular integration of communication platforms, and there is more situation of homogenization of content. People can perform a differentiated analysis of the audiences of OhPlay, add the radio stations according to their preferences, and increase the activity of the audiences. In addition, a full range of interactions can be carried out, and online radio activities can be combined with offline radio cultural festivals to allow as many audiences as possible to accept the information spread by MUZEN.

\subsection{Communication Audiences}

In the era of new media, target audiences are faced with more product choices, and audiences are gradually being diverted to different channels, making it difficult to bring them together. At the beginning, MUZEN RADIO positioned the audiences' age around 65 years old, which was single and inappropriate, resulting in poor initial sales. After adjustment, the audiences are in the range of 15-35 years old, and the sales volume exceeds 1 million. However, the design and brand culture of MUZEN RADIO are more attractive to female audiences, resulting in fewer purchases by male audiences. Therefore, different products can be designed according to the individual differences of different groups of people in the age range, and brand communication can be carried out on different media. [11]

At the same time, MUZEN RADIO also needs to pay attention to youthful interaction. In addition to the Radio Easter, it can also increase some campus activities to enhance interaction, which will help deepen the audiences' impression of MUZEN RADIO, and make the audiences recognize the brand from interaction and maintain loyalty to the brand.

\subsection{Communication Effect}

MUZEN RADIO has done a good job in the construction of brand culture. The anti-mainstream brand culture is deeply rooted in the hearts of the people, but the product quality still needs to be strengthened. When MUZEN promoted, the product was hand-made, but the sound effect was average when used, and it was difficult to keep the audiences loyal to the brand. The sound effect of the speaker is an important factor for the audiences to choose the products. Therefore, the MUZEN RADIO must first optimize the product quality and remain competitive among similar products. Secondly, strengthening the construction of brand culture can allow audiences to better understand the brand and enhance the effect of brand communication.

\section{CONCLUSION}

As one of the typical representatives of retro mini speakers, MUZEN RADIO has explored its own propagation path in the new media 
environment. It uses "social + word of mouth" for communication, focuses on the derivation of original content, all-round interaction and diversified communication, and regular brand interaction to cultivate audience loyalty. It has realistic reference and reference value for the brand communication of Chinese retro audio in China.

\section{AUTHORS' CONTRIBUTIONS}

Lu Wang wrote the paper, and Qiuyan Yun collected the data and edited the paper.

\section{REFERENCES}

[1] Yu Mingyang, Shu Yongping, About Brand Communication [J]. Journal of International Communication, 2002(03). (in Chinese)

[2] Li Hong, Brand Communication in the New Media Age [J]. Youth Journalist, 2018(08). (in Chinese)

[3] Tang Liping, The Trend of Brand Communication in the New Media Environment [J]. Journal of Chinese Youth Social Science, 2015, 34(02). (in Chinese)

[4] Wang Yingtong, The Influence of Brand Communication on Audience Culture Under the Background of New Media [J]. Market Modernization, 2018, (12). (in Chinese)

[5] Guan Xiaomin, Research on Brand Communication of B2B Enterprises in New Media Environment [J]. Media Form, 2019, 2(12). (in Chinese)

[6] Que Na, The New Concept of Brand Communication in the New Media Environment [J]. Youth Journalist, 2014(03). (in Chinese)

[7] Li Xiaonan, Analysis on Brand Communication in the New Media Environment [J]. Digital User, 2019, 25(3). (in Chinese)

[8] Liu Jianming, Zhang Wentian and Laswell's 5W Communication Mode [J]. The Press, 2014, (12). (in Chinese)

[9] Dong Qiaorong, Fan Economy in the Age of Information Network [J]. Gansu Argriculture, 2019, (11): 89-90. (in Chinese)

[10] Lai Shengqiang, Zheng Xiantao, Zhang Xuhui, Effect of Mianzi Psychology on the
Social Medial Users' EWOM Communication [J]. Journal of Information Resources Management, 2019, 9(2). (in Chinese)

[11] Xue Ke, Yang Changzheng, Yu Mingyang, Effect of Opinion Leader and Audience Orientation on Brand Communication on Comparative Research in Traditional and New Media Environment [J]. Soft Science, 2015, 29(2). (in Chinese) 